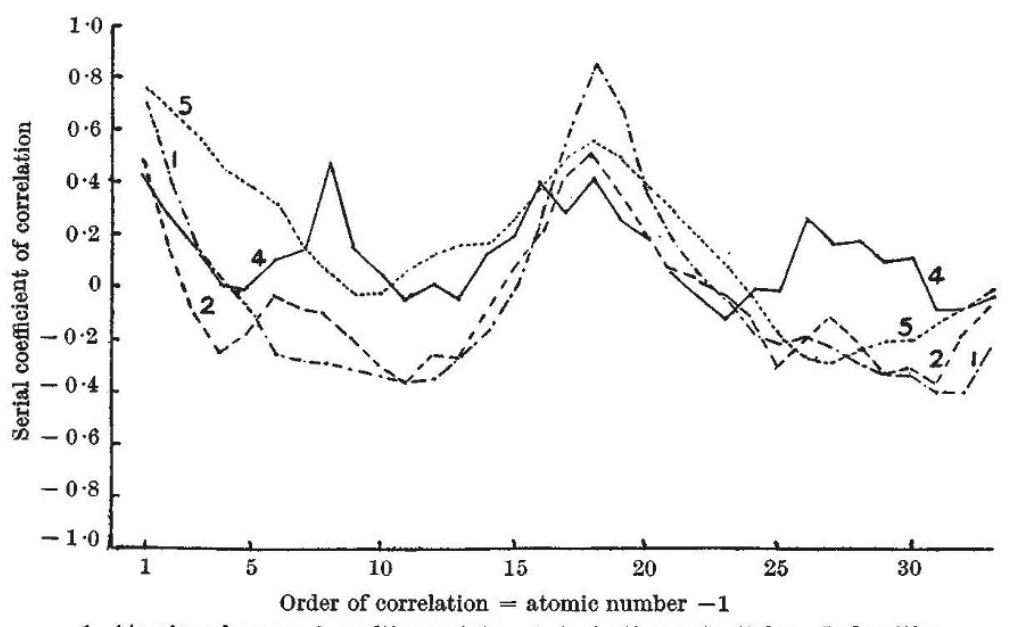

1 , Atomic volumes ; 2 , melting points ; 4 , ionization potentials ; 5 , densities

greater similarity to the chemical periodicity of the elements with its fundamental period of 8 .

Since the melting points (the temperature at which the atoms begin to leave the crystal lattice) and ionization potentials (the energy needed for removing an electron from the atom) have this in common, that they both refer to the point where lattice disorder sets in, and thus to the dynamic properties of matter, whereas atomic volumes, densities and compressibilities are properties of the static atom, it would seem that this difference is faithfully portrayed in the respective types of physical periodicity as revealed by the correlogram.

Investigations of this kind may afford an indication for linking more intimately than has been done so

It seemed of interest to inquire whether the generating mechanism of the physical periodicity was the same for all the physical properties to which it applied, or whether there existed characteristic differences between the periodicities of different physical properties. With the view of obtaining an answer to that question, some of the series were subjected to the statistical analysis called correlogram analysis ${ }^{1}$. The correlogram is the plot of the serial correlation coefficient against the order of correlation. For example, in the correlogram for atomic volume, the points are the correlation coefficients between the series of atomic volumes $1,2,3 \ldots n$ times apart in the series of the elements arranged according to their atomic numbers, plotted against the atomic number minus one.

The series subjected to the analysis were those of the atomic volumes (1), melting points (2), compressibilities (3), ionization potentials (4) and densities (5) of the first sixty elements. Series 1 to 4 are based upon Tables 2 and 16, Chapter 5 of "Physical Chemistry" by E. A. Moelwyn-Hughes; Series 5 is taken from Table 3, "Physical Chemistry" by Gucker-Meldrum, based upon the International Critical Tables, supplemented by Landolt-Börnstein tables. The first thirty-three serial correlation coefficients were calculated and plotted against the order of correlation; the curve for compressibilities has been left out of the diagram so as to avoid overcrowding.

On the basis of the correlogram it appears that what was hitherto called 'physical periodicity' has a different structure, that is, it represents sums of different cyclical components, for each of the physical characteristics under investigation. So far as the correlograms show similarities, it would seem that they can be classified into two types. The correlograms for atomic volumes, densities and compressibilities show a peak for the correlation of order 18 , and a trough for that of order 10 or very near it, which corresponds to the elements 19 (potassium) and 11 (sodium) respectively, and thus reveal a period of length 18, which corresponds to twice the fundamental period of the chemical periodicity of 8 , plus the first two elements of the table. The correlograms for melting points and ionization potentials, on the other hand, show, in addition to the period of length 18 (16), a smaller one with peaks at 8 and 26 , or very near them, and thus a far the mechanism of the various physical period. icities with the periodic system of the electrons constituting the shells and subshells of the atom.

C/o Paint Research Station,

G. HERDAN

Teddington, Middlesex. June 6.

${ }^{1}$ Kendall, M. G., "The Advanced Theory of Statistics", vol. 2 (1946).

\section{X-Ray Scattering by Thermal Vibrations in Crystals}

SIR C. V. RamaN, in his letter on "X-Rays and the Eigen-Vibrations of Crystal Structure"1, claims that the elastic vibrations cannot "give rise to any geometric diffraction pattern exhibiting an observable relationship to the structure of the crystal". The reasons given for this statement make it appear that he has fallen into Debye's original error, and supposes that the atomic vibrations are essentially independent, or are markedly dependent on the form of the crystal and on external boundary conditions. This would perhaps be the case for very small crystallites; for crystals of ordinary size the boundary conditions would involve second-order effects only, as they do in the case of Bragg reflexion. It should also be clearly understood that the change of frequency due to the X-ray 'Doppler effect' is so small that the modified wave-lengths still lie well within the width of the monochromatized $K \alpha_{1} \operatorname{lin} \theta$, assuming that to be the incident radiation; the change is of the order of 1 in $10^{5}$.

That the elastic vibrations do indeed give rise to a geometric diffraction pattern has been most conclusively proved by the excellent agreement between theory and experiment for metals such as sodium, lead and tungsten. The theory of eigen-vibrations can offer no explanation whatever of these experimental results, whereas the elastic vibration theory predicts them correctly in every detail ${ }^{2}$.

University College, KATHLEen LONSDALE

Gower Street,

London, W.C.1. July 15.

${ }^{1}$ Raman, C. V., Nature, 182, 23 (1948).

Jahn, H. A., Proc. Roy. Soc., A, 179, 320 (1942), Lonsdale, K., and Smith, H., Nature, 14*, 628 (1941); 149, 21 (1942). Lonsdale, K., Proc. Phys. Soc., j4, 314 (1942). 